\title{
Effects of Nitrogen Flow Rate on the Structural, Morphological and Optical Properties of TaN Thin Films Grown by the DC Magnetron Sputtered Technique
}

\author{
D.E. Cherfi ${ }^{a}$, M. Guemmaz ${ }^{a}$, M.E.H. Bourahli ${ }^{b}, *$ M.A. Oundfel ${ }^{c}$ And S. MaAbed $^{d}$ \\ ${ }^{a}$ DAC Laboratory, Faculty of Sciences, Ferhat Abbas University, Setif 1, Algeria \\ ${ }^{b}$ Precision Mechanic and Optic Institutes, Ferhat Abbas University, Setif 1, Algeria \\ ${ }^{c}$ Research Center in Semiconductor Technology for Energetic, Algiers, Algeria \\ ${ }^{d}$ Laboratory of Physics of Materials, Amar Telidji University, Laghouat, Algeria
}

(Received February 17, 2018; revised version July 16, 2019; in final form October 23, 2019)

\begin{abstract}
In this paper, the effect of nitrogen flow rates on the physical properties of thin TaN films deposited on glass and silicon substrates using reactive DC magnetron sputtering was experimentally studied. The structural and optical properties of the films were evaluated by X-ray diffraction, atomic force microscopy, and optical spectrophotometry. X-ray diffraction patterns revealed that the phase composition evolves from pure metallic $\alpha$-Ta body centred cubic to tantalum nitride $\delta$-TaN rock-salt structure. However, when the $\mathrm{N}_{2}$ flow rate exceeds $2 \mathrm{sccm}$, the films will show a poorly defined crystallization composed of mixture of fcc TaN phase and amorphous phases. Scanning electron microscopy and atomic force microscopy images revealed that the films deposited at high nitrogen flow rate possess smooth surface, good quality and lower roughness. The optical characterisation confirms that they are two groups of samples. The first have an metallic character with high reflectance and the second group are more transparent with an dielectric character with an absorption gap around $1.8 \mathrm{eV}$.
\end{abstract}

DOI: 10.12693/APhysPolA.136.849

PACS/topics: tantalum nitride, reactive magnetron sputtering, $\mathrm{N}_{2}$ flow rate, structural properties, optical properties

\section{Introduction}

Tantalum nitride $(\mathrm{TaN})$ thin films are a complex system. The TaN alloy forms a variety of phases depending on the deposition technique and growth conditions. Among the deposition techniques, there is the deposition by sputtering in a mixture of nitrogen and argon [1-5]. This technique has the advantage over several other ones that it can be easily scaled from small sized laboratory targets to industrial applications on large area substrates such as the coating of architectural glass [6]. The properties of $\mathrm{TaN}$ thin films obtained by this technique are extremely sensitive to the film's microstructure and deviations from stoichiometry. Furthermore, deposition rate, chemical composition, and crystalline structure of the deposited film depend on the ratio of $\mathrm{Ar}$ and $\mathrm{N}_{2}$ flow rates, chamber pressure, and power density [7, 8].

Numerous efforts have led to the determination of the properties of $\mathrm{TaN}$ versus $\mathrm{N}_{2}$ flow rate. Kang et al. [9] have studied the effects of varying $\mathrm{N}_{2}$ flow rates (0 to $20 \mathrm{sccm})$ on the structural properties of sputtered TaN films. They found that for a fixed $\mathrm{Ar}$ flow rate, the TaN films exhibit a single fcc structure. Valleti et al. [10] also investigated the structural

\footnotetext{
*corresponding author; e-mail: bourahlim@yahoo.fr
}

properties of $\mathrm{TaN}_{x}$ films by adding the nitrogen to argon gas ratio in the range $0.04-0.30$. The grown TaN thin films are found to contain mainly cubic$\mathrm{TaN}_{0.1}$, orthorhombic- $\mathrm{Ta}_{4} \mathrm{~N}$, orthorhombic- $\mathrm{Ta}_{6} \mathrm{~N}_{2.5}$, hexagonal- $\mathrm{Ta}_{0.8} \mathrm{~N}$ and cubic-TaN. The high hardness of $\approx 61.9 \mathrm{GPa}$ corresponds to orthorhombic $\mathrm{Ta}_{4} \mathrm{~N}$ phase. Tajima et al. [11] have also shown that the resistivity, film thickness, and crystallographic structure of TaN thin films could be controlled by changing $\mathrm{N}_{2}$ gas flow ratio, working pressure and sputtering power for the application to ultra large-scale integration (ULSI). Kwang et al. [12] studied optical properties and X-ray photoelectron spectroscopy of TaN thin films prepared by means of a reactive RF sputtering method on borosilicate glass substrates. They showed that the films prepared by $\mathrm{N}_{2}$ pressure $\geq 0.2$ mTorr presented an amorphous phase and the coefficient band gap increases with increasing $P\left(\mathrm{~N}_{2}\right)$ from 0.3 mTorr to 5.0 mTorr. In addition, they conclude that beyond $P\left(\mathrm{~N}_{2}\right) 5.0$ mTorr the films presented metastable phases similar to those of $\mathrm{Ta}_{2} \mathrm{O}$, which are closely related to the optical transparency. Aouadi et al. [13] have studied the effects of varying $\mathrm{N}_{2}$ flow rates from 1 to $4 \mathrm{sccm}$ on the structural and optical properties of $\mathrm{TaN}$ thin films. They report that the optical constants $\left(\varepsilon_{1}, \varepsilon_{2}\right)$ will be used in conjunction with real-time spectroscopic ellipsometry to monitor and control the growth of tantalum nitride films. Using ellipsometric spectroscopy 
and optical transmittance spectra, Mistrik et al. [14] have shown that $\mathrm{TaN}_{x}$ dielectric constants range from a metallic to a non-metallic character depending on substrate temperature, but non on the film thickness. They concluded that the substrate temperature dependence may be due to multiple phases in the $\mathrm{TaN}_{x}$ films. Matenoglou et al. [15] have found that the plasma energies of the $\delta$-TaN (111) deposited by ALD technique were to be 9.45 and $9.7[\mathrm{eV}]$ based on the experimental results and $a b$ initio calculations. The optical absorption bands were also observed around 1.9 and $7.3 \mathrm{eV}$. Wu et al. [16] added that the band gap TaN films on a $\mathrm{Si}(100)$ decrease with increasing films thicknesses. Constantin et al. [6] have studied the optical, decorative and structural properties taking into account the influence of reactive nitrogen gas flow variation. They showed that during the deposition procedures, different colors were obtained, from metallic-like to silver-like tones. They are directly correlated with the deposition conditions and the structure, respectively.

This paper attempts to provide a more detailed investigation regarding the effects the nitrogen flow rate on the structural and optical properties of tantalum nitride films deposited by DC magnetron sputtering on glass and Si (100) substrate.

\section{Experimental setup and procedure}

Tantalum nitride (TaN) thin films were prepared by direct current (DC) reactive magnetron sputtering from a polished metallic Ta target (99.96\% purity, 3 in diameter) using $\mathrm{N}_{2}$ as reactive gas. Single crystalline Si (100) wafer and glass were used as substrates. Both substrates were cleaned successively in ultrasonic baths of tetrachloro-ethylene (THF 50\%), acetone, and rinsing by deionized water, finally dried using $\mathrm{N}_{2}$ gas jet. Prior to film deposition, the system was pumped down to a pressure of about $5 \times 10^{-6}$ mbar. During deposition the pressure and the power were fixed at $2 \times 10^{-3} \mathrm{mbar}$ and $70 \mathrm{~W}$ respectively and an argon gas flow was kept constant at $8 \mathrm{sccm}$ whereas that of nitrogen gas flow was varied from 0 to $12 \mathrm{sccm}(1,2,4,8,12 \mathrm{sccm})$.

The structure was inferred from a PANalytical X'PertPRO MPD diffractometer with $\mathrm{Cu} \mathrm{K}_{\alpha}$ radiation $\lambda=1.5406 \AA$, using a $2 \theta$-step of 0.01 from 10 to $90^{\circ}$. The Scherrer Eq. (2.1) was used to estimate the average grain sizes of the crystallites

$$
D(2 \theta)=\frac{0.9 \lambda}{\beta \cos \theta},
$$

where $\beta$ is the half height width of the reflection peak at $2 \theta$ and $\lambda$ is the wavelength of the radiation. Microstructure was observed using a JEOL Hitachi S4700 SEM model scanning electron microscopy (SEM). Surface roughness and high magnification topography photographs of the deposited film were produced using Nanoscope III atomic force microscopy (AFM) in a non-contact mode. Changes in the surface roughness of the substrates after each deposit were monitored by obtaining $1 \times 1 \mu \mathrm{m}^{2}$ topographic for $2 \mathrm{D}$ images in $\mathrm{AC}$ mode using a silicon probe with $<10 \mathrm{~nm}$ tip radius and a cantilever nominal force constant of $40 \mathrm{~N} / \mathrm{m}$. For 3D surface morphology the films were scanned over a scan size of 5 and $5 \mathrm{~mm}$. Roughness values were calculated from the root mean square of the height amplitudes after the images were corrected for sample tilt. Measurements at five different sites on each substrate were performed.

Transmittance and reflectance spectra were recorded with a Cary 5000 scan UV-VIS-NIR spectrometer in the spectral range of 250 to $2500 \mathrm{~nm}$. In order to remove any possible organic contaminants, all samples were successively solicited in acetone, deionized water and dried in a nitrogen stream before measurement. The optical properties like as the dielectric function were deduced from analysis and fitting of the spectrometer data by RefFIT software [17].

\section{Results and discussion}

In this section, we may consider an experimental investigation to analyses the effect of nitrogen flow rates on the physical properties of thin TaN films deposited on glass and (100) silicon substrates using reactive DC magnetron sputtering. The optical properties were deduced from analysis and fitting of the spectrometer data.

\subsection{AFM and SEM results}

The variations in the surface roughness as a function of nitrogen flow are listed in Table I. Also 2D and 3D AFM images are shown in Fig. 1. Although the films were produced under the same conditions, a slight decrease in the root mean square (rms) roughness of the films is observed when the $\mathrm{N}_{2}$ flow rate increases.

SEM morphology (Fig. 1a) shows various topographies of the TaN films versus $\mathrm{N}_{2}$ flow rate. It can be seen that the pure Ta (S1) film shows a crystalline structure nondeformed columnar with quasi spherical granule shapes in agglomerated clusters.

The samples labeled S2 and S6 (corresponding to films made at 1 and $12 \mathrm{sccm}$ ), composed of nanocrystallites, exhibit better surface smoothness but contains irregular flakes of about $1 \mathrm{~nm}$ in thickness and a few hundred $\mathrm{nm}$ in width, randomly disposed. It is noted that the flakes becomes much larger and their number higher when $\mathrm{N}_{2}$ flow rate increases.

TABLE I

Roughness of the films of TaN/glass versus $\mathrm{N}_{2}$ flow rate

\begin{tabular}{c|c|c|c|c|c|c}
\hline \hline \multicolumn{1}{c|}{ Sample } & $\mathrm{S} 1$ & $\mathrm{~S} 2$ & $\mathrm{~S} 3$ & $\mathrm{~S} 4$ & $\mathrm{~S} 5$ & $\mathrm{~S} 6$ \\
\hline flow rate $\mathrm{N}_{2}[\mathrm{sccm}]$ & 0 & 1 & 2 & 4 & 8 & 12 \\
roughness $(\mathrm{rms})[\mathrm{nm}]$ & 9.2 & 4.3 & 3.7 & 2.9 & 2.9 & 2.4
\end{tabular}



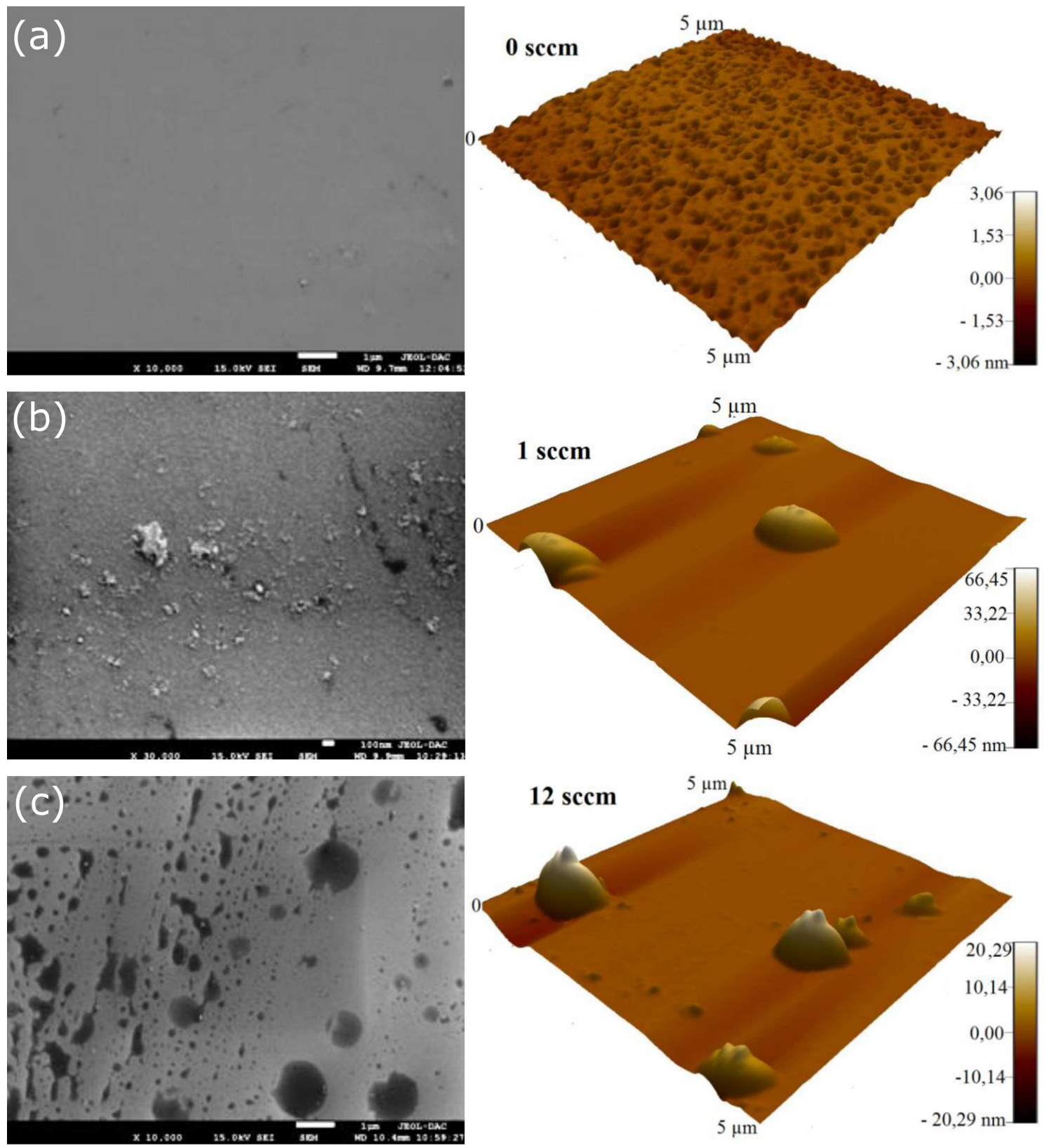

Fig. 1. Surface morphology of (S1, S2, S6) TaN films on glass substrate: (a) SEM, (b) AFM 2D image, (c) AFM 3D image.

\subsection{XRD analysis}

Figure 2a shows the dependence of $\mathrm{N}_{2}$ gas flow rate on XRD spectra for TaN thin films deposited on glass substrate. At $0 \mathrm{sccm}$, pure tantalum thin films were obtained. XRD analysis revealed the presence of the body-centered cubic (bcc) $\alpha$-Ta structure (JCPDS 04-0788) [18].

The addition of $\mathrm{N}_{2}$ has a direct impact on the film structure. Indeed, at a $\mathrm{N}_{2}$ flow rate of $1 \mathrm{sccm}$, the XRD spectrum permits to identify the main signature of the face-centered cubic (fcc) TaN phase also referred to as $\delta$ - $\mathrm{TaN}_{1-x}[19]$. The major peaks are characteristic of the rock-salt structure of the TaN phase (JCPDS 49-1283) and correspond to the (111) preferred orientation, (200), and (220) orientations, respectively. On increasing the $\mathrm{N}_{2}$ flow ratio to $2 \mathrm{sccm}$, the intensities of (111), (200) and (220) peaks shrinks corresponding to change in grain size. In addition, the peaks remarkably shift towards lower diffraction angle $2 \Theta$, which indicates an expansion of the unit cell and represents an increase in $d$-spacing along this plane. Aouadi et al. [13] reported that the TaN structure appears from $3 \mathrm{sccm}$ using a target of structure $\beta$-Ta. It appears that the nature of the target has a significant effect on the structure of film. 


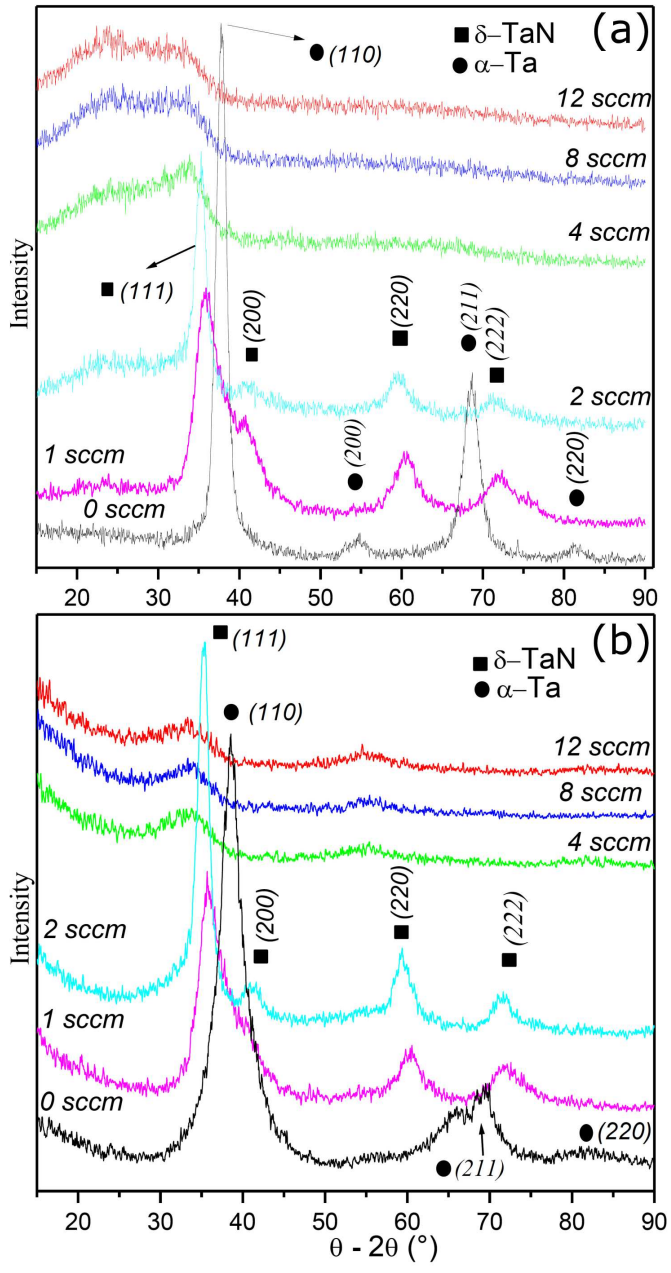

Fig. 2. Structural evolution (XRD patterns) of TaN deposited at various $\mathrm{N}_{2}$ flow rate on: (a) glass substrate, (b) $\mathrm{Si}(100)$ substrate.

Estimated crystallite sizes of TaN films

TABLE II

\begin{tabular}{c|c|c|c|c|c|c}
\hline \hline Sample & $\mathrm{S} 1$ & $\mathrm{~S} 2$ & $\mathrm{~S} 3$ & $\mathrm{~S} 4$ & $\mathrm{~S} 5$ & $\mathrm{~S} 6$ \\
\hline flow rate $\mathrm{N}_{2}[\mathrm{sccm}]$ & 0 & 1 & 2 & 4 & 8 & 12 \\
avg. cryst. size [nm] & 9.2 & 4.9 & 3.9 & 2.4 & 2.8 & 2.2
\end{tabular}

However, when the $\mathrm{N}_{2}$ flow rate exceeds 2 sccm, only dispersive peaks centered about $2 \Theta=34^{\circ}$ appear, which means the film have no obvious structure and a poorly defined crystallization. To determine the nature of this peak, the DRX spectrum (Fig. 2a) is compared with that of the film deposited on Si (100) substrate (Fig. 2b). In the latter one, we see clearly the (111) peak, while for the glass substrate there are both the broad peak with a little one at (111) position of film deposited on Si (100) tends to confirm that the broad peak is not an amorphous phase alone. The broadening peak could be attributed to the mixture of fcc TaN and amorphous phases and amorphous structure glass substrate [20].
In addition, the broadening of the diffraction peaks indicates that the samples are nanosized.

The average crystallites sizes estimated with the Scherrer equation using the peak (111) reflection (Table II) shows that the crystallite size of the S2 $(4.9 \mathrm{~nm})$ decreased drastically to $2.2 \mathrm{~nm}$ when a nitrogen flow rate of $12 \mathrm{sccm}$ (S6) is used. We conclude then that for low nitrogen flow rate (between 0 and $4 \mathrm{sccm}$ ), we have a well-defined crystalline structure with a $\langle 111\rangle$ texture. Whereas for higher nitrogen flow rate (more than $4 \mathrm{sccm}$ ), we observe a poorly defined crystallization with the presence of both a TaN (111) and amorphous phases. In fact, as we will see in the next section, there is a correlation between these structural properties and the optical ones.

\subsection{Optical properties}

Figure 3 shows the measured transmittance and reflectance spectra as a function of the wavelength for different compositions of TaN thin films on glass substrate.

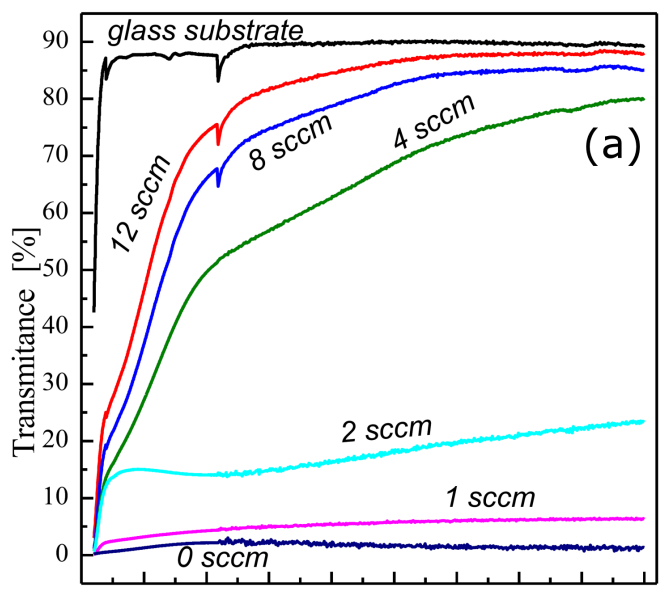

$250 \quad 500 \quad 750 \quad 1000125015001750200022502500$

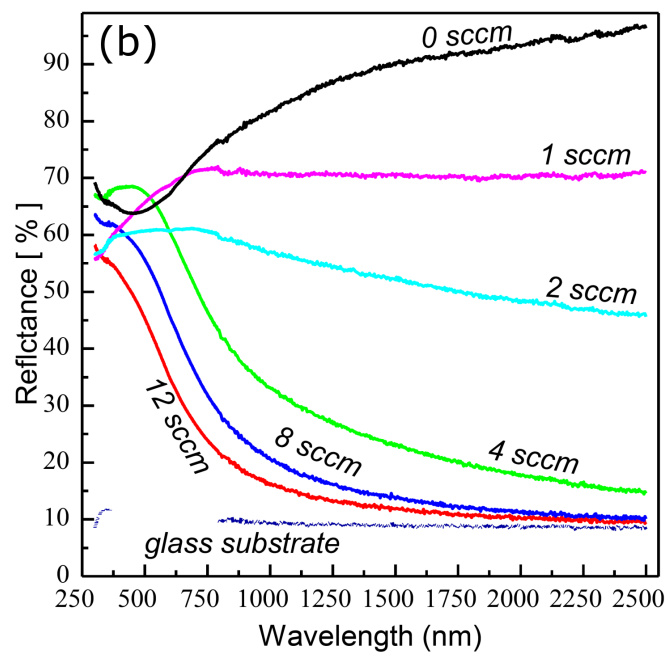

Fig. 3. Evolution of the optical wavelength for different $\mathrm{N}_{2}$ flow rate: (a) transmittance, (b) reflectance. 

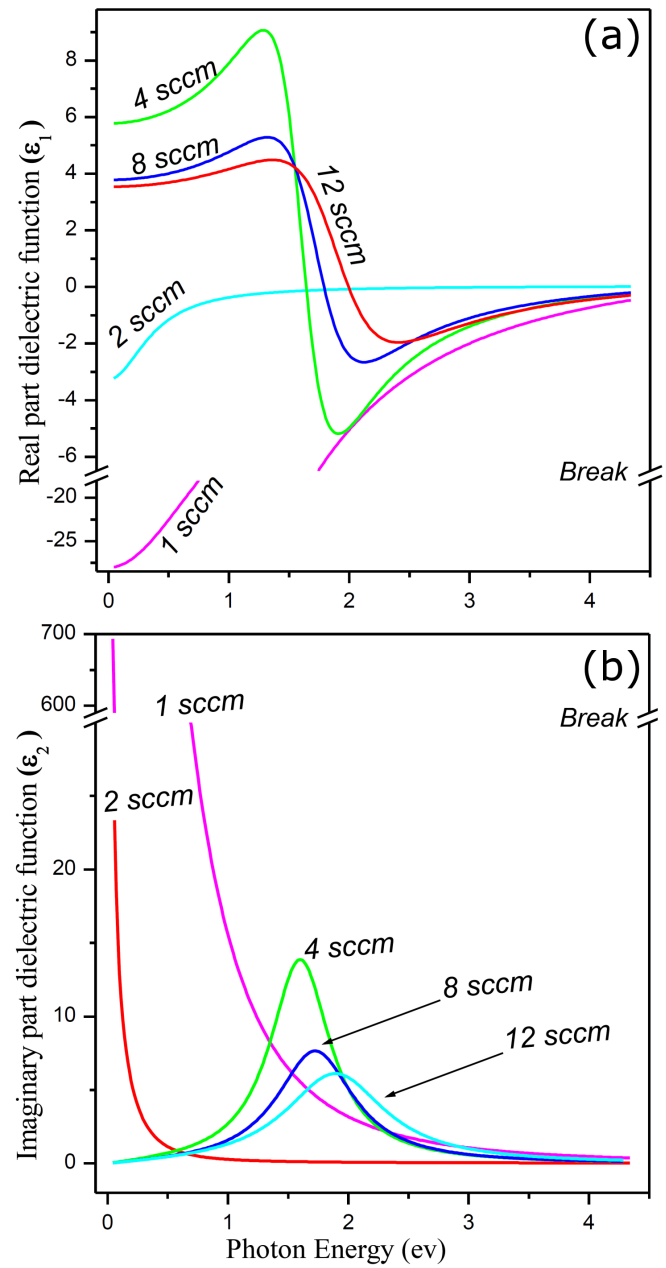

Fig. 4. Variation of dielectric function with photon energy: (a) real parts, (b) imaginary parts.

In Fig. 3a there is shown that the pure Ta film is non transparent in the region from $259 \mathrm{~nm}$ to $2500 \mathrm{~nm}$. It exhibits an absorbance of more than $20 \%$ in the region from $259 \mathrm{~nm}$ to $1500 \mathrm{~nm}$ and it is totally reflective beyond $1500 \mathrm{~nm}$ (Fig. 3b). The samples produced with 1 and $2 \mathrm{sccm}$ of gas flow presented a lower transmittance; this indicates a behavior that is similar to the one obtained with metallic samples. The addition of $\mathrm{N}_{2}$ tends to improve the optical transmittance. The coatings with highest values of the transmittance correspond to coatings with the higher value of nitrogen flow (12 sccm), varying from around $25 \%$ in the violet limit of the visible region of the spectrum, to around $75 \%$ in the red limit of the spectrum and reaches more than $85 \%$ for high wavelength. We note that the two peaks of absorption at 350 and $796 \mathrm{~nm}$ observed for 12 and $8 \mathrm{sccm}$ are caused by the substrate nature.

Figure 4 displays the calculated values of the real and imaginary parts of the complex dielectric function as a function of the photon energy for samples $\mathrm{S} 1-\mathrm{S} 6$. For both figures $4 \mathrm{a}-4 \mathrm{~b}$, one can make the following remarks. As we mentioned in the XRD analysis, the samples can be divided into two groups. Here also, one can see that for the low nitrogen flow rate (1 and $2 \mathrm{sccm}$ ) (Fig. 4a), the samples show good conductors behavior with plasma energy $E_{p}$ of about $5.5 \mathrm{eV}$ and $3.7 \mathrm{eV}$, respectively. This value is lower than the plasma energy reported by literature: for films $\delta$-TaN, $E_{p}=9.1 \mathrm{eV}$ obtained by ALD technique on Si (100) substrate and $\alpha$-Ta target [12], $E_{p}=8.1 \mathrm{eV}$ obtained by rf magnetron sputtering on $\mathrm{SiO}_{2}$ substrate by Mistrik [15], and $6.1 \mathrm{eV}$ by RDC technique on $\mathrm{Si}$ (100) and $\beta$-Ta target [10]. It seems that the deposition technique, substrate, $\mathrm{N}_{2}$ flow rate and the tantalum target type used have a direct effect on $E_{p}$.

For the second group (S4, S5 and S6), the samples exhibit a nonmetallic behavior. The corresponding imaginary part is a relatively narrow absorption curve (Fig. 4b); the absorption is the largest at the resonance frequency. Moreover, we see that the resonance frequency around $1.8 \mathrm{eV}$ monotonously increases as the nitrogen flow rate increases from 4 to $12 \mathrm{sccm}$. These results are in correspondence by the result reported by Matenoglou et al. [12].

We see thus that there is a clear correlation between the structural and optical properties: the samples with similar structural properties have similar behavior regarding the optical properties.

\section{Conclusion}

A new approach to the problem of nitrogen flow rate influence on the structural and optical properties of tantalum nitride films has been described.

This study has shown that increase in nitrogen flow lead to the decrease in film roughness. Certain homogeneity on the deposits was found at high nitrogen content. However, when the content exceeds $2 \mathrm{sccm}$, the structure showed only broad peak at $30^{\circ}$ attributed to the mixture of fcc of TaN phase and amorphous phases.

The plasma energy found is about $5.5 \mathrm{eV}$ for the films sputtered at $1 \mathrm{sccm}$. This value is lower than the plasma energy reported by literature. This is due to the effect of the deposition technique, the substrate, the $\mathrm{N}_{2}$ flow rate and the type of the tantalum target used.

The optical results show that we obtained a dielectric properties transparent sample for a $\mathrm{N}_{2}$ flow upper than 2 sccm and metallic properties samples for the lower flow values.

\section{References}

[1] W.H. Lee, J.C. Lin, C. Lee, Mater. Chem. Phys. 68, 266 (2001).

[2] N.K. Ponon, D.J.R. Appleby, Erhan Arac, P.J. King, Srinivas Ganti, K.S.K. Kwa, A. O'Neill, Thin Solid Films 578, 31 (2015).

[3] C.M. Koller, H. Marihart, H. Bolvardi, S. Kolozsvári, P.H. Mayrhofer, Surf. Coat. Technol. 347, 304 (2018). 
[4] Ran Li, Xiu-Zhi Duan, Xin Zhu, Yang Yang, DingBang Zhou, Zhi-Qing Li, Solid State Commun. 279, 34 (2018).

[5] D. Bernoulli, U. Müller, M. Schwarzenberger, R. Hauert, R. Spolenak, Thin Solid Films 548, 157 (2013).

[6] D.G. Constantin, C. Moura, D. Munteanu, Bull. Transilv. Univ. Brasov Ser. I Eng. Sci. 4, 59 (2011).

[7] J.C. Lin, G. Chen, C. Lee, J. Electrochem. Soc. 146 , 1835 (1999).

[8] Nishat Arshi, Junqing Lu, Chan Gyu Lee, Ben Heun Koo, Faheem Ahmed, JOM 66, 1893 (2014).

[9] Chang Seok Kang, H.J. Cho, Y.H. Kim, R. Choi, K. Onishi, A. Shahriar, J.C. Lee, J. Vac. Sci. Technol. B 21, 2026 (2003)

[10] K. Valleti, A. Subrahmanyam, V.J. Srikant, A.R. Phani, M. Passacantando, S. Santucci, J. Phys. D Appl. Phys. 41, 1 (2008).

[11] H. Tajima, N. Shiobara, H. Katsumata, S. Uekusa, J. Surf. Anal. 17, 247 (2011).

[12] B.L. Kwang, H.L. Kyung, J. Kor. Phys. Soc. 55, 966 (2009).
[13] S.M. Aouadi, M. Debessai, J. Vac. Sci. Technol. A 22, 1975 (2004)

[14] J. Mistrik, K. Takahashi, R. Antos, M. Aoyama, T. Yamaguchi, Y. Anma, Y. Fukuda, M.B. Takeyama, A. Noya, Z.-T. Jiang, S.M. Thurgate, G.V. Riessen, Thin Solid Films 455-456, 473 (2004).

[15] G.M. Matenoglou, L.E. Koutsokeras, Ch.E. Lekka, G. Abadias, S. Camelio, G.A. Evangelakis, C. Kosmidis, P. Patsalas, J. Appl. Phys. 104, 124907 (2008).

[16] Y.Y. Wu, M. Eizenberg, Mater. Chem. Phys. 101, 269 (2007).

[17] A. Kuzmenko, RefFIT software.

[18] P.N. Baker, Thin Solid Films 14, 3 (1972).

[19] H.O. Pierson, Handbook of Refractory Carbides and Nitrides, Properties, Characteristics, Processing and Applications, 1st ed., W. Andrew Publ., Noyes (NJ) 1996.

[20] X. Sun, E. Kolawa, J.S. Chen, J.S. Reid, M.A. Nicolet, Thin Solid Films 236, 347 (1993). 